\title{
Microscopic modeling of tunable graphene-based terahertz Landau-level lasers
}

\author{
Samuel Brem, ${ }^{1,2, *}$ Florian Wendler, ${ }^{2}$ and Ermin Malic ${ }^{1}$ \\ ${ }^{1}$ Department of Physics, Chalmers University of Technology, 41296 Gothenburg, Sweden \\ ${ }^{2}$ Institute of Theoretical Physics, Technical University Berlin, 10623 Berlin, Germany
}

(Received 21 April 2017; published 21 July 2017)

\begin{abstract}
In the presence of strong magnetic fields the electronic band structure of graphene drastically changes. The Dirac cone collapses into discrete nonequidistant Landau levels, which can be externally tuned by changing the magnetic field. In contrast to conventional materials, specific Landau levels are selectively addressable using circularly polarized light. Exploiting these unique properties, we propose the design of a tunable laser operating in the technologically promising terahertz spectral range. To uncover the many-particle physics behind the emission of light, we perform a fully quantum mechanical investigation of the nonequilibrium dynamics of electrons, phonons, and photons in optically pumped Landau-quantized graphene embedded in a high-quality optical cavity. The microscopic insights gained allow us to predict optimal experimental conditions to realize a technologically promising terahertz laser.
\end{abstract}

DOI: 10.1103/PhysRevB.96.045427

\section{INTRODUCTION}

The terahertz $(\mathrm{THz})$ regime of the electromagnetic spectrum can be exploited in a wide range of applications including medical diagnostics, atmosphere and space science, and security and information technology [1-4]. Although $\mathrm{THz}$ research has progressed significantly in the last 20 years, the transition from laboratory demonstration to practical environment remains challenging for the industry despite the tremendous potential of submillimeter waves. The largest challenge is the lack of adequate, frequency-tunable $\mathrm{THz}$ radiation sources. In 1986, Aoki proposed the design of Landau-level (LL) lasers exploiting the discreteness of LLs in two-dimensional electron gases [5]. Here, the energetic LL spacing and thus the possible laser frequency can be externally tuned through the magnetic field. The challenge in the realization of such a laser is to obtain a stable population inversion, i.e., a larger carrier occupation within an energetically higher LL. Since conventional semiconductors exhibit an equidistant spectrum of LLs, strong Coulomb scattering acts in favor of an equilibrium Fermi-Dirac distribution and strongly counteracts the buildup of a population inversion. In contrast, graphene as a two-dimensional material with a linear dispersion exhibits a nonequidistant LL separation offering entirely different conditions for many-particle processes $[6,7]$. Exploiting these remarkable properties of Landauquantized graphene, we propose an experimentally accessible scenario to achieve continuous wave lasing with tunable frequencies in the technologically promising terahertz spectral regime.

The nonequidistant arrangement of energy levels [8,9]

$$
\varepsilon_{l}=\operatorname{sgn}\{l\} \hbar v_{F} \sqrt{\frac{2 e_{0} B}{\hbar}|l|},
$$

combined with selection rules for circularly polarized light, allow us to selectively address individual inter-LL transitions. Here, the magnetic field $B$ is perpendicular to the graphene layer, $v_{F}$ denotes the Fermi velocity in graphene, and

\footnotetext{
*brem@chalmers.se
}

$l=\ldots,-2,-1,0,1,2, \ldots$ is the LL quantum number. Left (right) circularly polarized light, denoted $\sigma_{+(-)}$, exclusively induces transitions with quantum numbers $[10,11]|l| \rightarrow|l|+$ $(-) 1$. Thus, a linearly polarized optical pump field with an energy matching the transition $l=-2 \rightarrow+3$ simultaneously induces a population inversion between $l=+2$ and $l=+1$ as well as between $l=-1$ and $l=-2$ [cf. Fig. 1(a)].

To achieve lasing, graphene needs to be embedded in a highquality microcavity [13] with a resonator mode matching the energy difference between $l=+1$ and $l=+2$ [cf. Fig. 1(c)]. Thus, the population inversion leads to a multiplication of trapped cavity photons due to stimulated emission of $\mathrm{THz}$ photons via the transition $2 \rightarrow 1$ or, rather, $-1 \rightarrow-2$ [cf. Fig. 1(b)]. For continuous light amplification, radiative processes (optical pumping, emission of cavity photons) have to be complemented by nonradiative relaxation to establish a closed three-level laser system. In our exemplary pump scheme, efficient laser operation requires a fast depopulation of the lower laser level $l=+1$ and a repopulation of the pump level $l=-3$, which, e.g., can happen by a direct transition via the emission of optical phonons [cf. green arrow in Fig. 1(b)]. However, the interaction with phonons can concurrently assist the relaxation of carriers in the upper laser level as indicated by the red arrow in Fig. 1(b) and thereby counteract lasing. Since phonon-induced decay rates strongly depend on how well the transitions match the phonon energy, the efficiency of the laser scheme varies with the magnetic field, depending on how well the competing (laser cycle supporting and counteracting) LL transitions are in resonance with phonon modes.

In contrast to conventional materials, the nonequidistant spectrum of LLs in graphene efficiently quenches elastic Coulomb scattering processes, since they are allowed only if two LL transitions with the same energy are possible. Moreover, the proposed pump scheme induces an electronhole-symmetric carrier distribution, which further reduces the influence of Coulomb scattering, since each electron scattering process $l \rightarrow l^{\prime}$ in combination with the corresponding hole scattering $-l \rightarrow-l^{\prime}$ is exactly as strong as its respective inverse process.

While in previous studies [7,12] only the possibility of a population inversion between LLs in graphene has been 


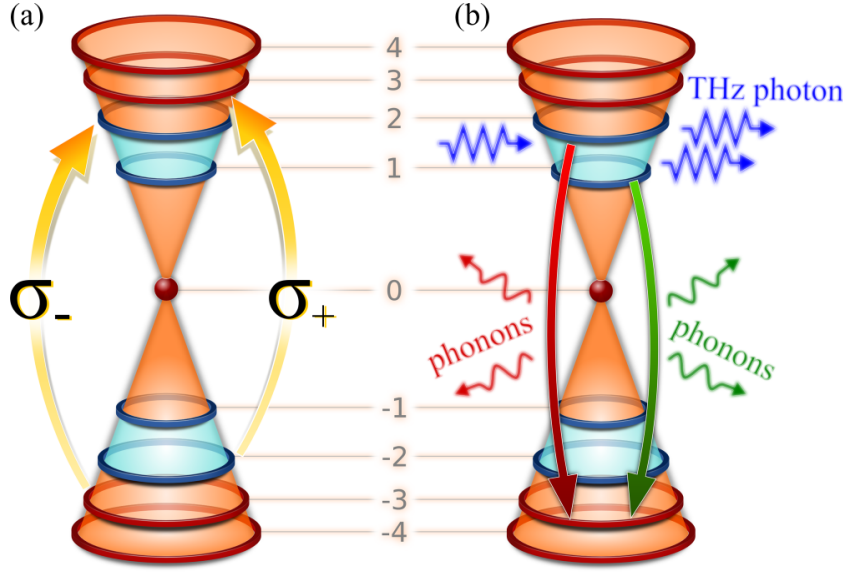

(c)

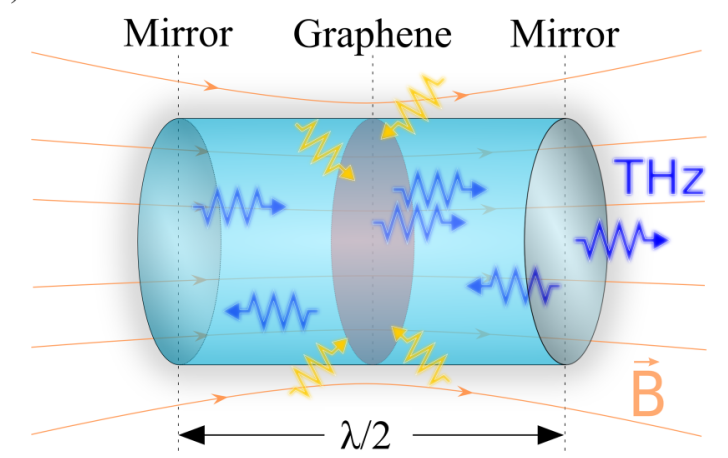

FIG. 1. Laser scheme. Sketch of the energetically lowest Landau levels in graphene embedded in an optical cavity. (a) The linearly polarized optical pump field induces transitions $l=-3 \rightarrow+2$ and $l=-2 \rightarrow+3$ (yellow arrows), which results in a population inversion between $l=+1$ and $l=+2$ in the conduction band and $l=-1$ and $l=-2$ in the valance band. (b) The emission of optical phonons can act in favor of the laser cycle (green arrows) or counteract the buildup of the population inversion (red arrows). (c) To achieve stimulated emission of photons, the system is embedded in an optical cavity.

theoretically predicted, in the current work we provide a fully quantum-mechanical modeling of the coupled dynamics of electrons, phonons, and photons in a Landau-level laser. The aim of our work is to provide profound insights into the complex interplay of pumping, stimulated and spontaneous emission of photons, and Coulomb- and phonon-induced nonradiative processes, allowing us to predict optimal conditions for lasing at different tunable magnetic fields. To model the laser dynamics, we use a quantum mechanical many-particle approach providing microscopic access to all relevant interaction processes in Landau-quantized graphene. The model allows us to provide a recipe for the realization of tunable THz LL lasers with optimal experimentally accessible conditions including magnetic field, cavity quality factor, and pump intensity.

\section{MICROSCOPIC MODEL}

Based on the density matrix formalism in second quantization [14-17] combined with tight-binding wave functions
$[8,18,19]$, we derive a set of luminescence Bloch equations:

$$
\begin{gathered}
\dot{\rho}_{l}(t)=2 \sum_{\mu l^{\prime}} \Re\left\{\left|g_{l^{\prime} l}^{\mu}\right|^{2} S_{l l^{\prime}}^{\mu}-\left|g_{l l^{\prime}}^{\mu}\right|^{2} S_{l^{\prime} l}^{\mu}\right\}+\sum_{l^{\prime}} P_{l l^{\prime}}\left(\rho_{l^{\prime}}-\rho_{l}\right) \\
+\Gamma_{l}^{\mathrm{in}}(t)\left(1-\rho_{l}\right)-\Gamma_{l}^{\mathrm{out}}(t) \rho_{l}, \\
\dot{n}_{\mu}(t)=8 N_{B} \sum_{l l^{\prime}}\left|g_{l l^{\prime}}^{\mu}\right|^{2} \Re\left\{S_{l^{\prime} l}^{\mu}\right\}-2 \kappa\left(n_{\mu}-n_{0}\right), \\
\dot{S}_{l l^{\prime}}^{\mu}(t)=i\left(\omega_{l l^{\prime}}+\omega_{\mu}+i \gamma_{l l^{\prime}}(t)+i \kappa\right) S_{l l^{\prime}}^{\mu}+n_{\mu}\left(\rho_{l^{\prime}}-\rho_{l}\right) \\
+\rho_{l^{\prime}}\left(1-\rho_{l}\right)+T_{l^{\prime}}^{\mu}-T_{l}^{\mu} .
\end{gathered}
$$

This system of differential equations describes the time development of the occupation probabilities $\rho_{l}(t)=\left\langle a_{l}^{\dagger} a_{l}\right\rangle(t)$ of LLs with quantum number $l$ and the number of photons $n_{\mu}(t)=\left\langle c_{\mu}^{\dagger} c_{\mu}\right\rangle(t)$ of the two cavity modes $\mu=\sigma \pm$, where $a_{l}^{\dagger}$ $\left(c_{\mu}^{\dagger}\right)$ is the electronic (photonic) creation operator. The carrier occupation is coupled to the number of photons via the photonassisted electron-hole correlation $S_{l l^{\prime}}^{\mu}(t)=\left\langle c_{\mu}^{\dagger} a_{l}^{\dagger} a_{l^{\prime}}\right\rangle(t)$, which is a measure of the probability of emitting a $\mu$ photon via the electronic LL transition $l^{\prime} \longrightarrow l$. Details about the derivation of the above equations can be found in the Appendix. Further, the derivation of all interaction coupling elements used can be found in Ref. [19].

Here, we briefly explain the different contributions in the dynamical equations and the appearing parameters. The interaction strength between electrons and cavity photons is determined by the coupling element $g_{l l^{\prime}}^{\mu}$ [cf. Eq. (A5), Appendix]. Furthermore, the photon generation rate is influenced by the number of emitters, which is given by the magnetic-field-dependent LL degeneracy $N_{B}=B A / \Phi_{0}$ corresponding to the number of magnetic flux quanta $\Phi_{0}=$ $h / e_{0}$ within the graphene layer of area $A$. We consider a finite cavity photon lifetime $(2 \kappa)^{-1}=Q / \omega_{\sigma \pm}$, which is given by the quality factor $Q$ and the magnetic-field-dependent photon frequency $\omega_{\sigma+}=\omega_{\sigma-}$. Therefore we account for photon losses due to cavity imperfections and laser light out-coupling, which leads to a decay of the photon number towards a thermal occupation $n_{0}$. The process of optical pumping enters the equations through the pump rate $P_{l l^{\prime}}$, which transfers carriers from $l$ to $l^{\prime}$. The explicit expression for the pump rate follows from a semiclassical treatment of the interaction of carriers with the optical pump field. Here, an adiabatic approximation for inter-LL coherences yields an analytical expression for the optical transition rates [cf. Eq. (A20) in the Appendix]. One finds that the pump rate is essentially given by the incident light intensity and contains energy conservation as well as the LL-specific selection rules as mentioned in Sec. I.

The carrier-carrier and carrier-phonon interactions are treated within a correlation expansion on a two-particle level [20], which leads to time- and energy-dependent in- and out-scattering rates $\Gamma_{l}^{\text {in/out }}(t)$. Those incorporate all electronelectron and electron-phonon scattering channels, i.e., all energy-allowed LL transitions, including time-dependent Pauli blocking terms. The explicit form of the scattering rates is given by (A13) in the Appendix. These particle conserving scattering rates complement the optical excitation via a redistribution of excited carriers along all LLs, leading to 
an ultrafast thermalization of the carrier system into a Fermi distribution.

The Coulomb interaction is calculated by taking into account many-particle screening in the random phase approximation according to the Lindhard formula $[8,11]$. The screening induced by the substrate is considered a dielectric background, where we have used the exemplary value of $\varepsilon_{b g}=3.3$ corresponding to a $\mathrm{SiC}$ substrate throughout this work. Within the relevant momentum regime, the energies of acoustic phonons are too low to induce inter-LL transitions. Therefore, carrier-phonon scattering is considered only for the dominant optical phonon modes $\Gamma \mathrm{TO}, \Gamma L O, \mathrm{KTO}$, and KLO [21,22] in a bath approximation [19], with $\epsilon_{\mathrm{KLO}}=151 \mathrm{meV}$, $\epsilon_{\text {Кто }}=162 \mathrm{meV}, \epsilon_{\text {ГLО }}=198 \mathrm{meV}$, and $\epsilon_{\text {Гто }}=192 \mathrm{meV}$.

We take into account finite lifetimes for many-particle correlations giving rise to a Lorentzian-type softening of the energy conservation within all transition rates. The width $\gamma_{l l^{\prime}}$ of the appearing Lorentz functions determines the broadening of LL transitions and is self-consistently calculated considering scattering- and impurity-induced dephasing [23-25]. Details of the self-consistent determination of the dephasing rates can be found in the Appendix. Recently predicted LL energy renormalization effects $[26,27]$ are neglected, since they are assumed to be small compared to the LL broadening $\gamma_{l l^{\prime}}$.

To prove whether coherent laser light is emitted from graphene, we also track the temporal evolution of the photon statistics via the second-order autocorrelation

$$
g_{\mu}^{(2)}(t)=\frac{\left\langle c_{\mu}^{\dagger} c_{\mu}^{\dagger} c_{\mu} c_{\mu}\right\rangle(t)}{\left\langle c_{\mu}^{\dagger} c_{\mu}\right\rangle(t)^{2}} .
$$

This quantity is a measure for the quantum mechanical intensity fluctuations of the emitted light [28]. Coherent laser light is characterized by $g^{(2)}(t)=1$, whereas $g^{(2)}(t)>1$ holds for thermal and $g^{(2)}(t)<1$ for nonclassical light. To calculate $g^{(2)}$ we consider the evolution of photon-photon and higher electron-photon correlations [as, for example, $T_{l}^{\mu}(t)=\Delta\left\langle c_{\mu}^{\dagger} a_{l}^{\dagger} a_{l} c_{\mu}\right\rangle(t)$ in Eq. (4)] up to the quadruplet level $[29,30]$ (cf. the Appendix).

\section{RESULTS}

The solution of the luminescence Bloch equations reveals the nonequilibrium dynamics of the electron distribution and the number of photons within the cavity, which provides a microscopic understanding of the switch-on characteristics of the Landau-level laser. In the following, we investigate the dynamics at room temperature and under the following experimentally accessible conditions: cavity cross-section area $A=1000 \mu \mathrm{m}^{2}$ determining the number of emitters [corresponding to the size of the graphene sheet; cf. Fig. 1(c)], cavity length fixed due to the resonance condition $L=\lambda_{\mu} / 2$, quality factor [31,32] $Q=5000$, and background screening $\varepsilon_{b g}=3.3$ (corresponding to a $\mathrm{SiC}$ substrate).

\section{A. Laser dynamics}

First, we study the laser dynamics at the fixed magnetic field $B=4 \mathrm{~T}$ and the pump intensity $I=20 \mathrm{~kW} / \mathrm{cm}^{2}$. Since in undoped graphene, the electron and hole populations within conduction and valence bands are fully symmetric, we focus on

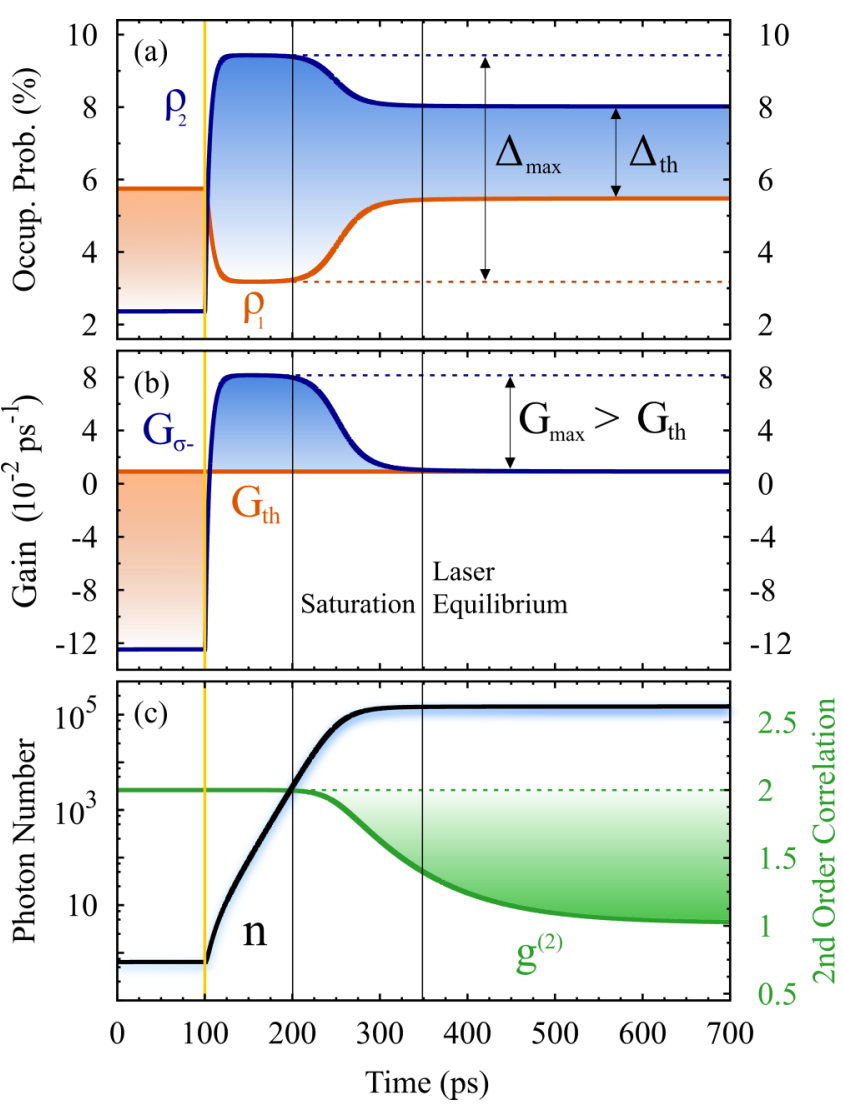

FIG. 2. Laser dynamics. (a) Time development of the occupation probabilities of the two laser levels in the conduction band $\rho_{2}$ and $\rho_{1}$ at $B=4 \mathrm{~T}$. The thermal electron population at $t=0$ is inverted (blue-shaded area) after the pump field is turned on (yellow line). Without stimulated emission, the inversion would stay at the indicated value $\Delta_{\max }$ (dashed lines). (b) Corresponding overall gain of the multilevel electronic system. (c) Evolution of the right circularly polarized photon number $n_{\sigma-}$ (logarithmic) and the second-order correlation $g_{\sigma-}^{(2)}$ (right axis). The population inversion induces an exponential increase in the photon number by stimulated emission. Due to the finite pump and relaxation rates, the population inversion is depleted with rising photon number (gain compression), resulting in a quasistationary threshold inversion $\Delta_{\text {th }}$. During the stable laser equilibrium the system emits coherent laser light characterized by the second-order correlation function $g_{\sigma-}^{(2)}=1$.

the electron dynamics in the following. Figure 2(a) shows the temporal evolution of the electron occupation probability of the two laser levels $l=+2$ and $l=+1$. Initially both occupations are at thermal equilibrium characterized by a Fermi-Dirac distribution with $\rho_{1}>\rho_{2}$. At $100 \mathrm{ps}$, the constant optical pump field is turned on, transferring carriers from $l=-3$ to $l=+2$, which leads to a population inversion with $\rho_{2}>\rho_{1}$ [cf. the blue-shaded region in Fig. 2(a)].

Phonon-induced relaxation of excited carriers counteracts the optical excitation mainly through the transitions $l=+2 \rightarrow$ -2 and $l=+2 \rightarrow-3$ [cf. red arrows in Fig. 1(b)]. At the chosen magnetic field of $B=4 \mathrm{~T}$ the transition $l=+1 \rightarrow$ -3 is in resonance with optical $\Gamma$ phonons. Therefore, the depopulation of $l=-3$ as result of optical pumping indirectly leads to a phonon-induced decrease in $\rho_{1}$. Coulomb-induced scattering is strongly suppressed due to the nonequidistant 
nature of the Landau quantization. The only efficient process is Auger scattering between equidistant LL transitions $2 \rightarrow$ 8 and $2 \rightarrow 0$. Shortly after the pulse is switched on, a quasiequilibrium between optical excitation and relaxation due to the emission of phonons is reached, resulting in a pump-induced population inversion $\Delta_{\max }$ [cf. Fig. 2(a)]. The resulting photon gain generated from the pumped electronic system is shown in Fig. 2(b), which is discussed further below.

Including an optical cavity, the number of photons increases exponentially via stimulated emission, once a population inversion is established. Figure 2(c) shows the time evolution of the right circularly polarized photon number $n_{\sigma-}$. For about 100 ps the population inversion stays stable despite the growing photon avalanche. However, after approximately 200 ps it slowly decreases, reflecting the so-called gain compression, which results from the finite pump and relaxation rates. The occupation of the upper laser level $\rho_{2}$ decreases, since the stimulated emission of photons via $l=+2 \rightarrow+1$ breaks the balance between pumping and phonon relaxation. Similarly, the finite lifetime of $l=+1$ leads to an accumulation of carriers, resulting in an enhanced $\rho_{1}$ [cf. Fig. 2(a)]. After approximately $350 \mathrm{ps}$, a new quasiequilibrium is reached that is characterized by a reduced threshold population inversion $\Delta_{\text {th }}$. At that point, the photon generation via $l=+2 \rightarrow+1$ and photon losses due to cavity imperfections or off-resonant absorption via other LL transitions compensate each other and the number of photons remains constant [Fig. 2(c)].

The time evolution of the photon number, Eq. (3), can be simplified by considering the static limit of Eq. (4) under negligence of spontaneous emission and higher order electron-photon correlations. Then the photon dynamics can be written as

$$
\dot{n}_{\mu}(t) \approx\left(G_{\mu}(t)-2 \kappa\right) n_{\mu} .
$$

Here, we have introduced the gain coefficient

$$
G_{\mu}(t)=8 \pi N_{B} \sum_{l l^{\prime}}\left|g_{l l^{\prime}}^{\mu}\right|^{2}\left(\rho_{l}(t)-\rho_{l^{\prime}}(t)\right) \mathcal{L}_{\gamma_{l l^{\prime}}+\kappa}\left(\omega_{l^{\prime} l}-\omega_{\mu}\right)
$$

including the coupling with all LL transitions $l \rightarrow l^{\prime}$. The coefficient is given by the carrier occupation difference and the Lorentzian $\mathcal{L}_{\gamma}(\omega)$ determining the LL width $\gamma$. As a result, $\mathrm{LL}$ transitions, which are slightly detuned from the laser frequency ( $\Delta \epsilon \sim \hbar \gamma$ ), can reabsorb cavity photons and thereby lower the gain generated by the inverted LL transition. Figure 2(b) shows the temporal evolution of the gain coefficient. To be able to generate photons, the overall gain has to exceed the threshold gain $G_{\text {th }}=2 \kappa$, which is given by the cavity photon lifetime $\tau_{\text {photon }}=(2 \kappa)^{-1}$. Moreover, to enter the lasing regime, the gain has to be stabilized with pumping and phonon relaxation to hold it above the threshold value during the growing photon avalanche. After a certain number of photons is reached, the gain is compressed to the threshold value. Due to the finite pump and relaxation efficiency, the light amplification saturates until it compensates the cavity losses, which characterizes the stable laser equilibrium. Here, the internal quantum efficiency, i.e., the effective fraction of emitted laser photons per absorbed pump photon, is about $3.5 \%$. This comparatively low value results from the Coulomb- and phonon-induced relaxation of the upper laser level and from the parasitic absorption of cavity photons by the off-resonant transitions $l=$ $2 \rightarrow 3, l=3 \rightarrow 4$, etc. However, the quantum efficiency can be optimized, since the loss via unwanted scattering processes could be reduced by using dielectric substrates, which significantly screen the Coulomb interaction. Furthermore, lower operation temperatures would increase the Pauli blocking and therefore suppress the unwanted phonon relaxation.

Finally, to describe the statistics of the emitted photons, we calculate the second-order correlation function $g^{(2)}$ [cf. the right $y$ axis in Fig. 2(c)]. Initially before the optical pumping, we find $g^{(2)}=2$ characterizing photons in thermal equilibrium. Once a population inversion is reached, the number of photons increases due to stimulated emissions, and $g^{(2)}$ approaches the value 1 , characterizing coherent laser light.

\section{B. Tunability of the laser frequency}

A crucial advantage of the LL laser is the straightforward tunability of its frequency, since the spacing between LLs is adjustable through the magnetic field. However, as discussed in the last section, the efficiency of a laser scheme essentially depends on phonon-induced nonradiative processes stabilizing the gain during stimulated emission. The probability for the emission of a phonon significantly changes with the energetic separation $\hbar \omega_{l l^{\prime}}$ of the involved levels, since it depends on how well the transition matches the phonon energy $\hbar \Omega_{v \mathbf{q}}$. Consequently, the fast emission of $\Gamma$ phonons via $l=+1 \rightarrow$ -3 , complementing the laser cycle at $B=4 \mathrm{~T}$, will become inefficient if the magnetic field is changed. In this section, we investigate the tunability of a graphene-based LL laser from a microscopic perspective. We vary the magnetic field widely and then answer the question in what frequency range the proposed laser scheme works. For a single tunable laser device, a change in the magnetic field would need to be complemented by a tunable mid-IR pump laser in the range of 5-9 $\mu \mathrm{m}$ and a dynamic cavity of adjustable length in the range of $20-40 \mu \mathrm{m}$.

To obtain insight into the competing nonradiative processes, we apply the same conditions at each magnetic field by holding the pump transition rate constant at $P_{0}=1.2 \times$ $10^{-2} \mathrm{ps}^{-1}$ (corresponds to the scenario at $B=4 \mathrm{~T}$ and $I_{\text {pump }}=$ $20 \mathrm{~kW} / \mathrm{cm}^{2}$ ), only exciting carriers via $-3 \rightarrow 2$ and $-2 \rightarrow 3$. Moreover, the length $L$ of the cavity is adjusted to the $B$ dependent resonance condition $L=\lambda_{\mu} / 2=\pi c \hbar /\left(\varepsilon_{2}-\varepsilon_{1}\right)$.

Figure 3 shows the temporal evolution of the overall gain [Fig. 3(a)], the photon number [Fig. 3(b)], and the second-order correlation function $g^{(2)}$ [Fig. 3(c)] for the $\sigma_{-}$polarization within the technologically relevant magnetic-field range. Complex interplay of the multiple phonon resonances as well as Coulomb-scattering processes gives rise to a remarkable magnetic-field dependence, exhibiting alternating $B$ ranges with drastically different laser dynamics. More precisely, we distinguish four zones, called I-IV, where lasing takes place.

In analogy to the discussion in the last section, we now describe the temporal evolution of the system, allowing us to explain the different behavior in the four lasing zones and to identify the dominating many-particle mechanisms assisting or harming the laser scheme. As in the last section, the thermal equilibrium at $t=0$ is pumped to an intermediate quasiequilibrium at $t=100 \mathrm{ps}$. Only if the achieved gain [blue 
(a)

(b)
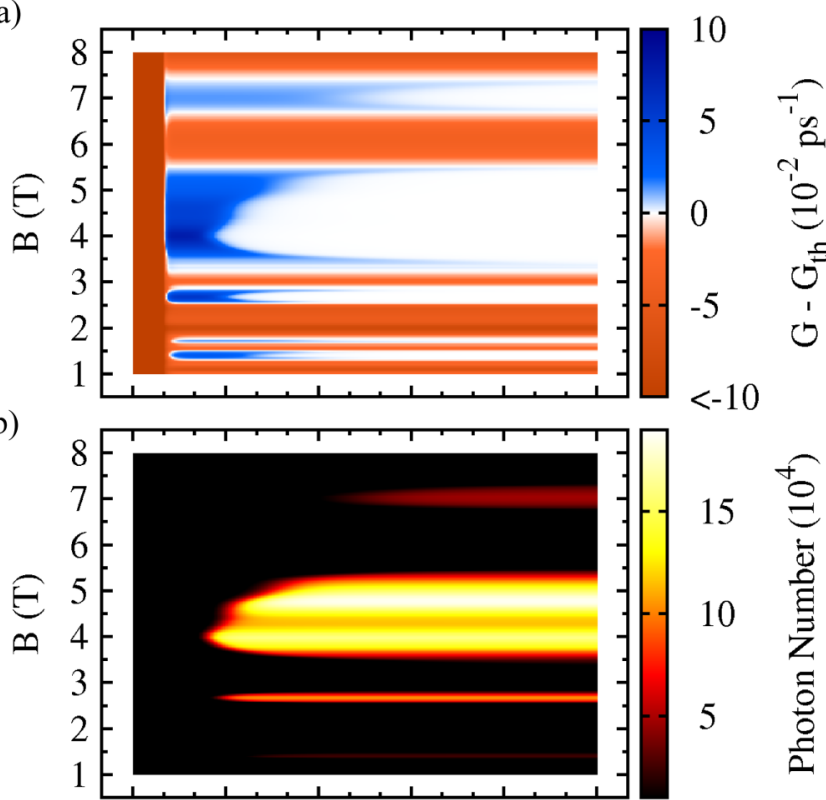

(c)

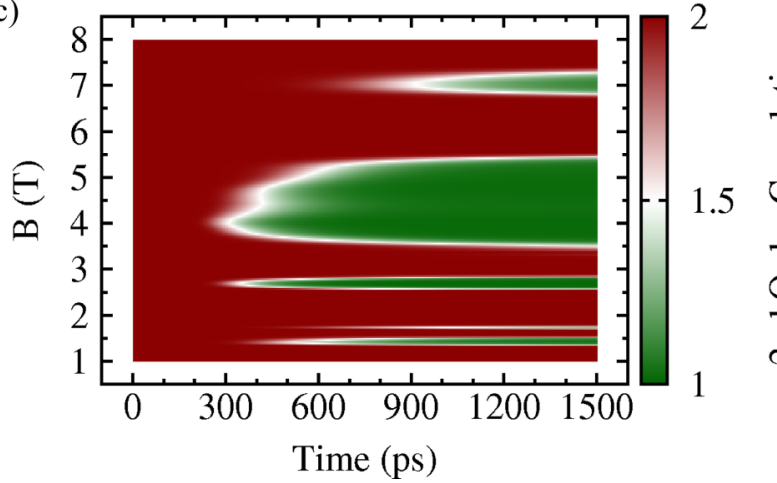

FIG. 3. Laser frequency tunability. Time development of (a) the gain, (b) the photon number, and (c) the second-order correlation function $g^{(2)}$ for technologically relevant magnetic fields at a constant pump rate. The emission of optical phonons can either support or counteract the laser operation, which leads to a remarkable magnetic-field dependence of the laser dynamics. There are four regimes (labeled I-IV), where the achieved gain is large enough to generate a significant number of photons and to produce coherent $\mathrm{THz}$ radiation characterized by $g^{(2)}=1$.

areas in Fig. 3(a)] significantly exceeds the cavity losses given by $G_{\text {th }}$ can stimulated emission induce a photon avalanche resulting in an exponential increase in emitted photons [cf. bright areas in Fig. 3(b)] characterized by $g^{(2)}=1$, describing coherent laser light [cf. green areas in Fig. 3(c)].

The appearance of distinct magnetic-field zones with varying laser characteristics can be well understood by examining the $B$ dependence of the efficiency of carrier-phonon scattering processes. The rate of a phonon-induced LL transition $l \rightarrow l^{\prime}$ can be described in analogy with Fermi's golden rule,

$$
\begin{aligned}
\sigma_{l l^{\prime}}= & \frac{2 \pi}{\hbar^{2}} \sum_{\nu \mathbf{q}}\left|G_{l l^{\prime}}^{\nu \mathbf{q}}\right|^{2}\left(N_{\nu \mathbf{q}} \mathcal{L}_{\gamma_{l l^{\prime}}}\left(\omega_{l l^{\prime}}+\Omega_{\nu \mathbf{q}}\right)\right. \\
& \left.+\left(N_{\nu \mathbf{q}}+1\right) \mathcal{L}_{\gamma_{l l^{\prime}}}\left(\omega_{i j}-\Omega_{\nu \mathbf{q}}\right)\right)
\end{aligned}
$$

where we sum over all phonon modes $v$ with momentum $\mathbf{q}$ weighted by the square of the electron-phonon matrix element [19] $G_{l l^{\prime}}^{v \mathbf{q}}$ and the phonon occupations $N_{v \mathbf{q}}$.

In Fig. 4, we compare the magnetic-field dependence of the maximum gain (left axis) and the equilibrium photon number (right axis) [Fig. 4(a)] and the phonon-induced transition rates for the most relevant scattering channels [Fig. 4(b)]. Hereby, the laser assisting transitions are represented by blue shading in Fig. 4(b), whereas counteracting decay rates are shaded red. First, we discuss the behavior for the magnetic fields $B>2 \mathrm{~T}$, where the LLs involved in the laser scheme are sufficiently far away from each other to allow direct transition via the emission of phonons. Apparently, the lasing zones, labeled I-IV, are characterized by strong resonances for supporting channels [cf. Fig. 4(b)] such as $1 \rightarrow-3$ in zones II and III. This transition allows efficient continuous-wave (cw) operation since carriers can perform cycles in a three-level system and also enhances the maximum gain by lowering $\rho_{1}$. As a result, the gain exceeds the threshold, which allows the generation of a large number of photons (cf. upper panel). In zones III and IV the $1 \rightarrow-1$ channel assists the cw operation by depleting the lower laser level and, additionally, coupling the two-laser transition in conduction and valence bands. The simultaneous resonance of both mentioned supporting channels explains the maximal photon number at approximately $B=4.8 \mathrm{~T}$.

The lasing zones are separated by regimes (shaded gray) where the pumped gain stays below the threshold [orangeshaded areas in Fig. 4(a)]. Here, phonon relaxation processes dominate, which strongly counteract lasing by depleting the population inversion, e.g., via direct relaxation of the upper laser level $2 \rightarrow-2,-3$, explaining the lack of lasing between zone I and zone II as well as between zone II and zone III. Additionally, resonances with LLs, which are not directly involved in the laser scheme, also play an important role, as the relaxation $8 \rightarrow 1$, e.g., at $B=6 \mathrm{~T}$. As a consequence of Auger scattering $2 \rightarrow 8$ (in addition to $2 \rightarrow 0$ ), the pump process leads to an unwanted accumulation of carriers in $l=8$. Therefore, an efficient phonon relaxation $8 \rightarrow 1$ causes an indirect pumping of the lower laser level, counteracting the population inversion between zone III and zone IV.

At low magnetic fields, $B=1-2 \mathrm{~T}$, the LLs involved in the laser scheme are too close to allow direct phonon relaxations. The resulting accumulation of pumped carriers gives rise to enhanced Auger scattering into higher lying LLs, such as the already mentioned process involving $l=8$ or the equivalent process for the lower laser level $1 \rightarrow 4$ in parallel with $1 \rightarrow 0$. Hence, phonon resonances involving $l= \pm 4, \pm 8$ determine the behavior at low magnetic fields by providing indirect relaxation channels. For magnetic fields below $1 \mathrm{~T}$, neighboring LLs begin to strongly overlap, so that the applied theoretical approach is no longer valid.

Another interesting aspect of the laser dynamics is the behavior at the borders of the four lasing zones. Although the pumped gain initially exceeds the threshold, e.g., at $B \sim 6.5 \mathrm{~T}$, we do not observe a stable laser operation (no photons in equilibrium). Here, the laser supporting nonradiative processes are too slow, so that the gain saturates or even falls below the threshold, before the avalanche of stimulated emission generates a significant number of photons. As a result, a population inversion does not guarantee lasing. 


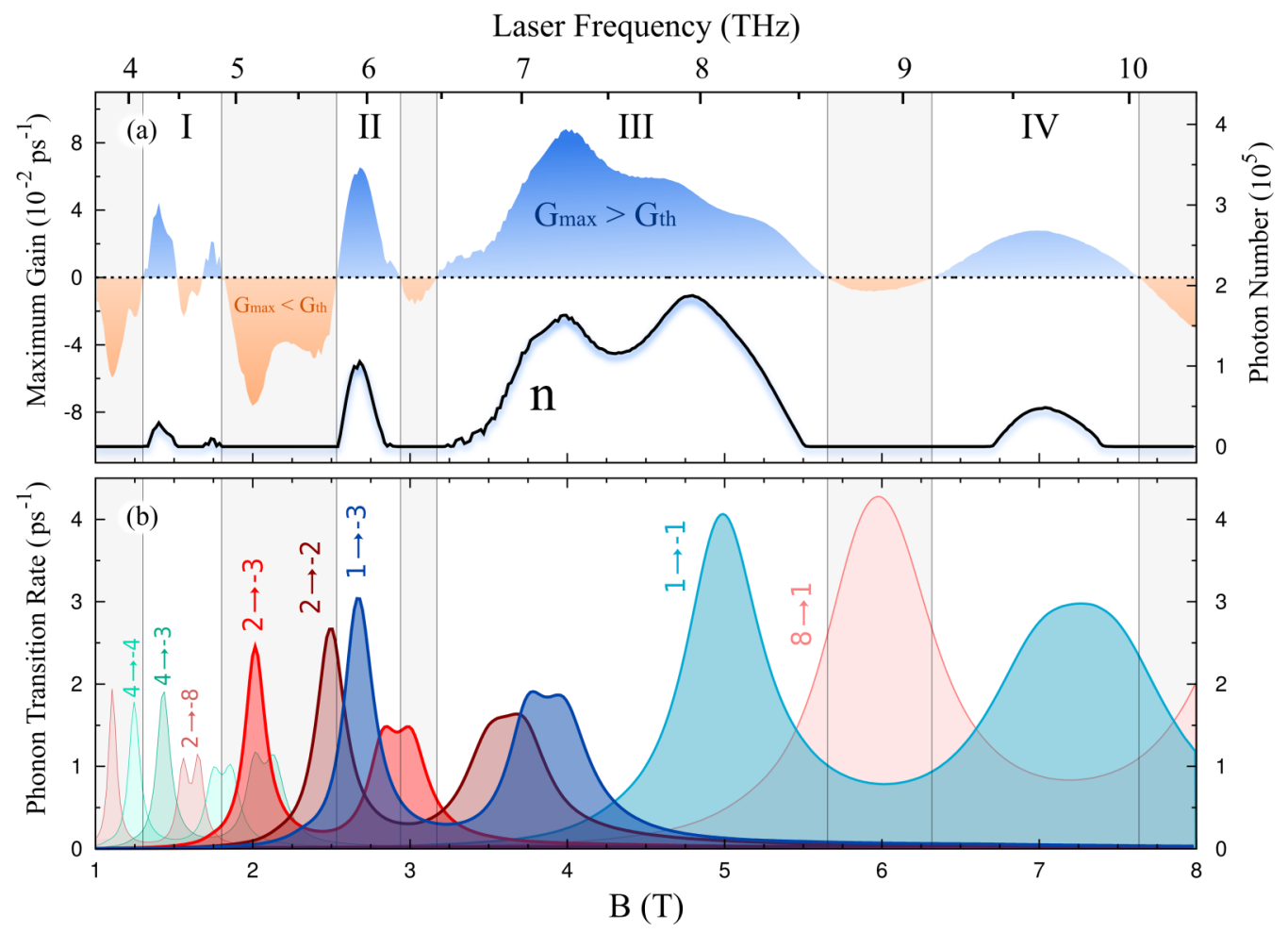

FIG. 4. Interplay of phonon resonances. (a) Magnetic-field dependence of the maximum pumped gain (left axis) and the number of photons at equilibrium (right axis). A significant number of photons (black curve) is generated only when the pumped gain exceeds the threshold (blue-shaded parts of the upper curve). (b) Phonon-induced transition rates for the most relevant channels. The distinguished laser zones from Fig. 3 are determined by the efficiency of carrier-phonon scattering channels that either support a closed laser cycle or counteract the population inversion.

To sum up, the proposed LL laser is widely tunable due to the broadening of LLs allowing multiple nonradiative relaxation pathways supporting the laser cycle. At a reasonable pump rate, we find four distinct magnetic field zones in which the laser works, generating coherent radiation within the technologically desired $\mathrm{THz}$ range.

\section{Optimal conditions}

So far, we have investigated the laser dynamics at a fixed optical pump rate, cavity quality factor, and temperature. Here, we vary these experimentally accessible quantities, aiming at optimal conditions for lasing. We assume again that the Landau-level laser is driven by a tunable pump laser and has a cavity which changes the length in line with the magnetic field in order to stay in full resonance with the laser transition, i.e., $L=\pi c \hbar /\left(\epsilon_{2}-\epsilon_{1}\right)$. Furthermore, when changing the magnetic field, the pump frequency is adjusted to match the transition energy of $-3 \rightarrow 2$. Since the distance between LLs and also their broadening increase with the magnetic field, the pump rate $P \propto I /\left(\omega^{2} \gamma\right)$, i.e., the number of excited carriers per time, decreases when the incident intensity $I$ of the pump field remains constant.

Figure 5(a) illustrates the number of photons within quasiequilibrium as a function of the pump intensity and the magnetic field at room temperature and $Q=5000$. In black areas, the pump intensity is too low to establish lasing. The pronounced line between dark and bright areas denotes the threshold intensity as a function of the magnetic field. The threshold line shows a general upward trend, owing to the decrease in the pump rate. Furthermore, it exhibits maxima and minima, which can again be ascribed to the efficiency of laser supporting and counteracting phonon resonances, as discussed in the last section. While phonon-induced LL transitions $1 \rightarrow-3$ [cf. Fig. 4(b)] act in favor of a low threshold at 2.5 and $4 \mathrm{~T}$, the counteracting phonon-induced transitions $2 \rightarrow-2,-3$ give rise to maxima in the threshold line at 2 and $3 \mathrm{~T}$. The phonon resonance $8 \rightarrow 1$ at $B=6 \mathrm{~T}$ results in a threshold intensity of several hundred $\mathrm{kW} / \mathrm{cm}^{2}$, since it indirectly couples $l=2$ and $l=1$, which counteracts the selective excitation of the upper laser level.

Assuming that the cavity photon lifetime $1 /(2 \kappa)$ is solely limited due to laser light out-coupling, the overall output intensity can be estimated as $I_{\text {out }}=2 \kappa \sum_{\mu} \hbar \omega_{\mu} n_{\mu} / A$. Figure 6(a) in the Appendix shows the input-output curve for three characteristic magnetic fields, $B=1.5,3$, and $4 \mathrm{~T}$. Our calculations reveal that even in the chosen exemplary model, based on one single graphene layer as the gain medium, $\mathrm{THz}$ output powers of several $\mathrm{W} / \mathrm{cm}^{2}$ are achievable. The low input/output ratio [Fig. 6(a)], in the range of $10^{-4}-10^{-5}$ mainly results from the low absorption efficiency of our lasing setup, where only a small fraction $(\sim 1 \%)$ of the incident pump light is absorbed by the graphene layer. In a real laser device, the pump absorption efficiency could be significantly increased by using stacks of separated graphene layers and reusing unabsorbed pump light via mirrors. The presence of 


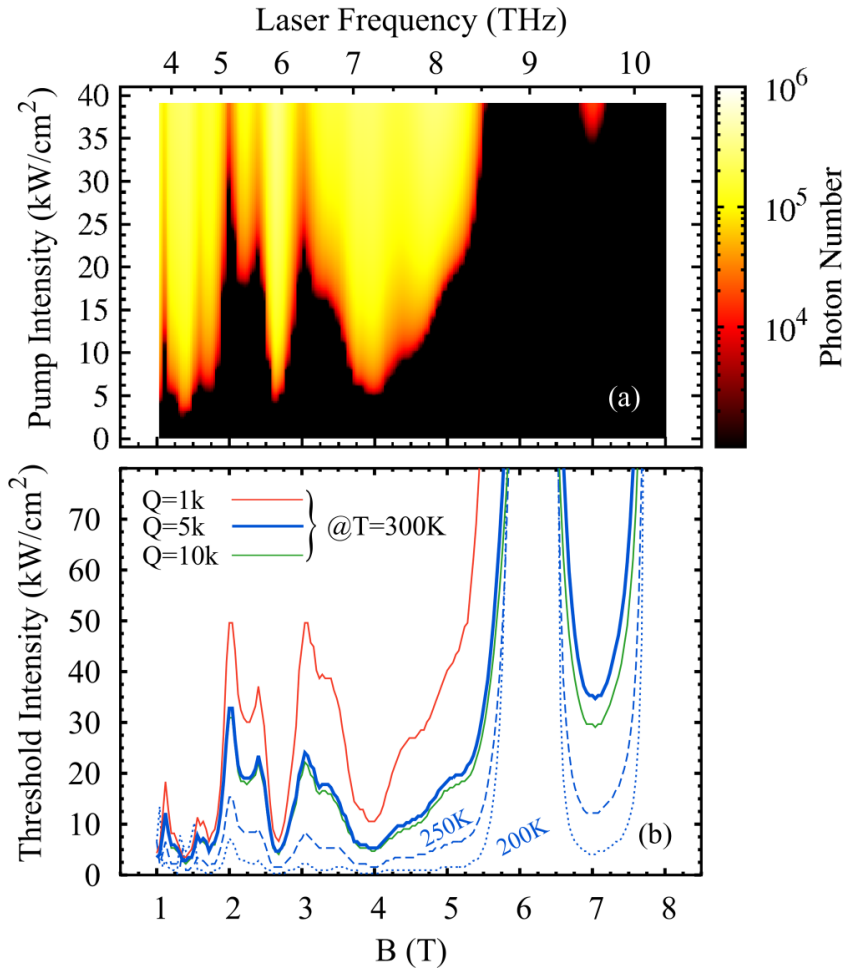

FIG. 5. Laser threshold. (a) Photon number at quasiequilibrium as a function of the magnetic field $B$ and pump intensity. For each $B$, there is a minimum pump intensity required to achieve lasing. The $B$ dependence of the laser threshold and the photon generation efficiency reflect the interplay of laser cycle supporting and counteracting phonon processes. (b) Magnetic-field dependence of the threshold pump intensity for different cavity quality factors $Q$ and temperatures $T$. High qualities and low temperatures act in favor of a lower laser threshold.

several graphene layers would additionally lead to an effective increase in the number of emitters per cavity cross section, which would yield an enhanced matter-light coupling and therefore would significantly improve the internal quantum efficiency.

Furthermore, the laser characteristics can be modified by improving the experimental conditions. Figure 5(b) shows the magnetic field dependence of the threshold pump intensity for different cavity quality factors $Q$ (at room temperature) and different temperatures $T$ (at the fixed $Q=5000$ ). In general, high quality factors and low temperatures lead to an overall decrease of the laser threshold. Since the $Q$-factor is a measure for the photon lifetime, it directly scales the threshold gain $G_{\text {th }}=\left(\tau_{\text {photon }}\right)^{-1}=\omega_{\text {photon }} / Q$. However, for $Q \longrightarrow \infty$, the minimum pump intensity still has to be sufficient enough to induce a positive gain, namely to invert the thermal occupation of the laser levels and to compensate the off-resonant absorption by other LL transitions. Hence, we observe a saturation behavior for high quality factors. Finally, a cooling of the system would further be beneficial to obtain lower threshold intensities, since the lower the thermal occupation of $l=1$, the fewer carriers need to be pumped into $l=2$ to achieve a population inversion. Additionally, low temperatures decrease the efficiency of counteracting phonon emissions due to an increased Pauli blocking, which benefits the internal quantum efficiency of the laser mechanism.

One can conclude that provided the pump power is sufficient, the proposed laser design is in principle tunable over a broad spectral range at room temperature. When applying a constant pump intensity of $40 \mathrm{~kW} / \mathrm{cm}^{2}$, the Landau-level laser can be continuously tuned in the range $4-8.5 \mathrm{THz}$ by applying magnetic fields of about $1-5.5 \mathrm{~T}$.

In conclusion, we predict a strategy to achieve coherent terahertz laser emission exploiting the unique properties of graphene in magnetic fields. Based on a microscopic and fully quantum-mechanical study of the coupled electron, phonon, and photon dynamics in optically pumped Landau-quantized graphene coupled to an optical cavity, we show that the emission of coherent terahertz radiation can be obtained under feasible experimental conditions. Provided the high-quality cavity is adequate and the pump power sufficient, the proposed laser scheme works in the frequency range between 4 and $8.5 \mathrm{THz}$. The presented work provides a concrete recipe for the experimental realization of tunable graphene-based terahertz laser systems.

\section{ACKNOWLEDGMENTS}

This project received funding from the European Union's Horizon 2020 research and innovation program under Grant Agreement No. 696656. Furthermore, we acknowledge financial support from the Swedish Research Council (VR) and the Deutsche Forschungsgemeinschaft (DFG) through SPP 1459. Finally, we thank A. Knorr (TU Berlin) and S. Winnerl (Helmholtz-Zentrum Dresden-Rossendorf) for inspiring discussions on Landau-level lasers.

\section{APPENDIX}

\section{Theoretical approach}

\section{a. Many-particle Hamilton operator}

The temporal evolution of electrons in Landau-quantized graphene coupled to a set of photon and phonon modes is determined by the many-particle Hamilton operator

$$
H=H_{\mathrm{el}}+H_{\mathrm{ph}}+H_{\mathrm{pt}} .
$$

The electronic part reads

$$
\begin{aligned}
H_{\mathrm{el}}= & H_{0, \mathrm{el}}+H_{\mathrm{el}-\mathrm{el}}+H_{\mathrm{el}-\mathrm{l}} \\
= & \sum_{i} \varepsilon_{i} a_{i}^{\dagger} a_{i}+\frac{1}{2} \sum_{i j k l} V_{i j k l} a_{i}^{\dagger} a_{j}^{\dagger} a_{k} a_{l} \\
& -i \hbar \frac{e_{0}}{m_{0}} \sum_{i j} \mathbf{M}_{i j} \cdot \mathbf{A}(t) a_{i}^{\dagger} a_{j}
\end{aligned}
$$

and is constituted by the electronic creation and annihilation operators $a_{i}^{\dagger}$ and $a_{i}$. Here the compound index $i=\left(l_{i}, m_{i}, s_{i}, \xi_{i}\right)$ determines the electronic state $[8,19]$, containing the Landaulevel index $l=\ldots,-2,-1,0,1,2, \ldots$ and the quantum number $m=0,1, \ldots, N_{B}-1$, which can be associated with the position of the cyclotron orbits in the graphene plane of surface $A\left[N_{B}=A e_{0} B /(2 \pi \hbar)\right.$ is the number of magnetic flux quanta within the graphene plane], the spin $s= \pm 1 / 2$, and 
the valley index $\xi= \pm 1$. We include the free contribution of carriers with eigenenergies $\varepsilon_{i}$ (cf. the text), the carrier-carrier interaction determined by the Coulomb matrix element $V_{i j k l}$, and a semiclassical carrier-light coupling, which is given by the optical matrix element $\mathbf{M}_{i j}=\langle i|\nabla| j\rangle$ and the local vector potential $\mathbf{A}(t)$. The elementary charge and the electron mass are denoted $e_{0}$ and $m_{0}$, respectively. The tight-binding expressions of the electronic eigenenergies and eigenfunctions and all matrix elements can be found in our review article on Landau-quantized graphene [19]. The semiclassical carrierlight coupling is used to describe the interaction with the optical pump field, whereas the light of the laser mode is treated fully quantum mechanically.

The phonon (photon) part of the Hamiltonian denoted by the subscript 'ph' ('pt') is given by

$$
\begin{aligned}
H_{\mathrm{ph}} & =H_{0, \mathrm{ph}}+H_{\mathrm{el}-\mathrm{ph}} \\
& =\sum_{\nu \mathbf{q}} \hbar \Omega_{\nu \mathbf{q}} b_{\nu \mathbf{q}}^{\dagger} b_{\nu \mathbf{q}}+\sum_{i j v \mathbf{q}} G_{i j}^{v \mathbf{q}} a_{i}^{\dagger} a_{j}\left(b_{\mathbf{q} v}+b_{-\mathbf{q} v}^{\dagger}\right), \\
H_{\mathrm{pt}}= & H_{0, \mathrm{pt}}+H_{\mathrm{el}-\mathrm{pt}} \\
= & \sum_{\mu} \hbar \omega_{\mu} c_{\mu}^{\dagger} c_{\mu}-i \hbar \sum_{i j \mu}\left(g_{i j}^{\mu} a_{i}^{\dagger} a_{j} c_{\mu}-g_{i j}^{\mu *} a_{j}^{\dagger} a_{i} c_{\mu}^{\dagger}\right)
\end{aligned}
$$

and includes phononic (photonic) creation operators $b_{\nu \mathbf{q}}^{\dagger}\left(c_{\mu}^{\dagger}\right)$, corresponding to the mode $v(\mu)$ and the phonon momentum q. It consists of a free part given by the phonon (photon) frequency $\Omega_{\nu \mathbf{q}}\left(\omega_{\mu}\right)$ and an interaction part including the carrier-phonon (carrier-photon) matrix element $G_{i j}^{\mathbf{v q}}\left(g_{i j}^{\mu}\right)$.

The electron-photon Hamiltonian can be deduced from the semiclassical electron-light coupling by quantizing the vector potential $\mathbf{A}$ and expanding it in normal modes. Hence, the electron-photon matrix element is given by

$$
g_{i j}^{\mu}=\frac{e_{0}}{m_{0}} \sqrt{\frac{\hbar}{2 \epsilon_{0} V \omega_{\mu}}} \mathbf{M}_{i j} \cdot \mathbf{e}_{\mu},
$$

with the normalized polarization vector of the photon mode $\mathbf{e}_{\mu}$ and the quantization volume $V$, which in the case of a laser is equal to the volume of the cavity.

\section{b. Equations of motion}

We evaluate the Heisenberg equation of motion $i \hbar \partial_{t}\langle\mathcal{O}\rangle=$ $\langle[\mathcal{O}, H]\rangle$ to determine the temporal evolution of the occupation probabilities of electronic eigenstates $\rho_{i}=\left\langle a_{i}^{\dagger} a_{i}\right\rangle$ and the photon numbers $n_{\mu}=\left\langle c_{\mu}^{\dagger} c_{\mu}\right\rangle$. To prove whether coherent laser light is emitted from graphene, we also track the temporal evolution of the photon statistics via the second-order correlation function $g^{(2)}$, which for zero delay time is given by

$$
g_{\mu}^{(2)}(t)=\frac{\left\langle c_{\mu}^{\dagger} c_{\mu}^{\dagger} c_{\mu} c_{\mu}\right\rangle(t)}{\left\langle c_{\mu}^{\dagger} c_{\mu}\right\rangle(t)^{2}}=2+\frac{h_{\mu}(t)}{n_{\mu}(t)^{2}} .
$$

Coherent laser light (Poisson statistics) is characterized by $g^{(2)}(t)=1$, whereas $g^{(2)}(t)>1$ holds for thermal and $g^{(2)}(t)<$ 1 for nonclassical light [28]. To calculate $g^{(2)}$ we need to consider the evolution of the photon-photon correlation $h_{\mu}(t)=\left\langle c_{\mu}^{\dagger} c_{\mu}^{\dagger} c_{\mu} c_{\mu}\right\rangle^{c}(t)$. To this end, we calculate all relevant electron-photon-correlations up to the quadruplet level [29,30], thus including equations for $T_{i}^{\mu}(t)=\left\langle c_{\mu}^{\dagger} a_{i}^{\dagger} a_{i} c_{\mu}\right\rangle^{c}(t)$ and $U_{i j}^{\mu}(t)=\left\langle c_{\mu}^{\dagger} c_{\mu}^{\dagger} a_{i}^{\dagger} a_{j} c_{\mu}\right\rangle^{c}(t)$. Carrier-carrier and carrierphonon correlations beyond doublets are neglected. We obtain the set of coupled differential equations

$$
\begin{gathered}
\frac{d}{d t} \rho_{i}=2 \sum_{\mu, j} \Re\left\{\left|g_{j i}^{\mu}\right|^{2} S_{i j}^{\mu}-\left|g_{i j}^{\mu}\right|^{2} S_{j i}^{\mu}\right\}+\sum_{j} P_{i j}\left(\rho_{j}-\rho_{i}\right)+\Gamma_{i}^{\mathrm{in}}\left(1-\rho_{i}\right)-\Gamma_{i}^{\mathrm{out}} \rho_{i}, \\
\frac{d}{d t} n_{\mu}=2 \sum_{i j}\left|g_{i j}^{\mu}\right|^{2} \Re\left\{S_{j i}^{\mu}\right\}-2 \kappa_{\mu}\left(n_{\mu}-n_{\mu}^{0}\right), \\
\frac{d}{d t} S_{i j}^{\mu}=i\left(\omega_{i j}+\omega_{\mu}+i \kappa_{\mu}+i \gamma_{i j}\right) S_{i j}^{\mu}+\rho_{j}\left(1-\rho_{i}\right)-n_{\mu}\left(\rho_{i}-\rho_{j}\right)-T_{i}^{\mu}+T_{j}^{\mu}, \\
\frac{d}{d t} T_{i}^{\mu}=-\left(2 \kappa_{\mu}+\gamma_{i i}\right) T_{i}^{\mu}+2 \sum_{j} \Re\left\{\left|g_{j i}^{\mu}\right|^{2} U_{i j}^{\mu}-\left|g_{i j}^{\mu}\right|^{2} U_{j i}^{\mu}\right\}+2 \sum_{j} \Re\left\{\left|g_{j i}^{\mu}\right|^{2} S_{i j}^{\mu}\left(n_{\mu}+1-\rho_{i}\right)-\left|g_{i j}^{\mu}\right|^{2} S_{j i}^{\mu}\left(n_{\mu}+\rho_{i}\right)\right\}, \\
\frac{d}{d t} U_{i j}^{\mu}=i\left(\omega_{i j}+\omega_{\mu}+3 i \kappa_{\mu}+i \gamma_{i j}\right) U_{i j}^{\mu}-2\left|g_{j i}^{\mu}\right|^{2}\left(S_{i j}^{\mu}\right)^{2}-h_{\mu}\left(\rho_{i}-\rho_{j}\right)-2 n_{\mu}\left(T_{i}^{\mu}-T_{j}^{\mu}\right)+2\left(1-\rho_{i}\right) T_{j}^{\mu}-2 \rho_{j} T_{i}^{\mu}, \\
\frac{d}{d t} h_{\mu}=4 \sum_{i j}\left|g_{i j}^{\mu}\right|^{2} \Re\left\{U_{j i}^{\mu}\right\}-4 \kappa_{\mu} h_{\mu},
\end{gathered}
$$

where we have rescaled $S_{i j}^{\mu} \longrightarrow g_{j i}^{\mu} S_{i j}^{\mu}$ and $U_{i j}^{\mu} \longrightarrow g_{j i}^{\mu} U_{i j}^{\mu}$ for simplicity. Further, $\omega_{i j}=\left(\varepsilon_{i}-\varepsilon_{j}\right) / \hbar$ denotes the electronic transition frequency and the finite photon lifetime $\left(2 \kappa_{\mu}\right)^{-1}=Q / \omega_{\mu}$ accounts for cavity losses, which are determined by the cavity quality factor $Q$. The Coulomb and phonon interactions are treated within the second-order Born-Markow approximation [17], which gives rise to the scattering rates $\Gamma_{i}^{\text {in/out }}=\Gamma_{i}^{\text {in/out,el }}+\Gamma_{i}^{\text {in/out,ph }}$, with

$$
\begin{gathered}
\Gamma_{i}^{\mathrm{in}, \mathrm{el}}=\frac{2 \pi}{\hbar^{2}} \sum_{a b c} V_{a b c i}\left(V_{c i a b}-V_{i c a b}\right) \rho_{a} \rho_{b}\left(1-\rho_{c}\right) \mathcal{L}\left(\gamma_{a c}+\gamma_{b i}, \omega_{a c}+\omega_{b i}\right), \\
\Gamma_{i}^{\mathrm{out}, \mathrm{el}}=\frac{2 \pi}{\hbar^{2}} \sum_{a b c} V_{a b c i}\left(V_{c i a b}-V_{i c a b}\right)\left(1-\rho_{a}\right)\left(1-\rho_{b}\right) \rho_{c} \mathcal{L}\left(\gamma_{a c}+\gamma_{b i}, \omega_{a c}+\omega_{b i}\right),
\end{gathered}
$$




$$
\begin{gathered}
\Gamma_{i}^{\mathrm{in}, \mathrm{ph}}=\frac{2 \pi}{\hbar^{2}} \sum_{j v \mathbf{q}}\left|G_{i j}^{\nu \mathbf{q}}\right|^{2} \rho_{j}\left(N_{\nu \mathbf{q}} \mathcal{L}\left(\gamma_{i j}, \omega_{j i}+\Omega_{\nu \mathbf{q}}\right)+\left(N_{\nu \mathbf{q}}+1\right) \mathcal{L}\left(\gamma_{i j}, \omega_{j i}-\Omega_{\nu \mathbf{q}}\right)\right), \\
\Gamma_{i}^{\mathrm{out}, \mathrm{ph}}=\frac{2 \pi}{\hbar^{2}} \sum_{j \nu \mathbf{q}}\left|G_{i j}^{v \mathbf{q}}\right|^{2}\left(1-\rho_{j}\right)\left(N_{\nu \mathbf{q}} \mathcal{L}\left(\gamma_{i j}, \omega_{i j}+\Omega_{\nu \mathbf{q}}\right)+\left(N_{\nu \mathbf{q}}+1\right) \mathcal{L}\left(\gamma_{i j}, \omega_{i j}-\Omega_{\nu \mathbf{q}}\right)\right) .
\end{gathered}
$$

Within a bath approximation the phonon number $N_{v \mathbf{q}}=$ $\left\langle b_{\nu \mathbf{q}}^{\dagger} b_{\nu \mathbf{q}}\right\rangle$ is fixed to the thermal occupation (Bose-Einstein statistics). Moreover, phonon scattering is considered only for the dominant optical phonon modes ГTO, ГLO, KTO and KLO [21,22], with $\epsilon_{\mathrm{KLO}}=151 \mathrm{meV}, \epsilon_{\mathrm{KTO}}=162 \mathrm{meV}$, $\epsilon_{\Gamma L O}=198 \mathrm{meV}$, and $\epsilon_{\text {Гто }}=192 \mathrm{meV}$ (Einstein approximation), since the energies of acoustic phonons are too low within the relevant momentum regime to induce inter-LL transitions.

The energy conservation is softened due to the Lorentzian broadening

$$
\mathcal{L}(\gamma, \omega)=\frac{1}{\pi} \frac{\gamma}{\gamma^{2}+\omega^{2}},
$$

whose width is given by the dephasing $\gamma_{i j}$, which is selfconsistently determined [17] considering impurity and manyparticle scattering. It reads

$$
\begin{aligned}
\gamma_{i j} & =\gamma_{\text {imp }}+\gamma_{i j}^{\mathrm{el}}+\gamma_{i j}^{\mathrm{ph}} \quad \text { with } \\
\gamma_{i j}^{\mathrm{el} / \mathrm{ph}} & =\frac{1}{2} \sum_{k=i, j}\left(\Gamma_{k}^{\mathrm{in}, \mathrm{el} / \mathrm{ph}}+\Gamma_{k}^{\mathrm{out}, \mathrm{el} / \mathrm{ph}}\right) .
\end{aligned}
$$

Since the scattering rates $\Gamma_{i}$ themselves depend on the dephasing, they are determined iteratively starting with $\gamma_{i j}=$ $\gamma_{\text {imp. }}$.

The disorder contribution to the equation of motion is derived within a self-consistent Born approximation, following the approach of Shon and Ando [23,24]. We assume [19]

$$
\gamma_{\mathrm{imp}}=\frac{v_{F}}{l_{B} \sqrt{A_{\mathrm{imp}}}}=v_{F} \sqrt{\frac{e_{0} B}{\hbar A_{\text {imp }}}},
$$

where $A_{\text {imp }}$ denotes a dimensionless parameter characterizing the scattering strength of the impurity potential $[23,24]$. Since this parameter is not accessible in experiments, we assume the impurity parameter $A_{\mathrm{imp}}=420$, since the corresponding broadening of $2.5 \mathrm{meV}$ at $B=4 \mathrm{~T}$ is in good agreement with experimental studies of linewidths in absorption spectra [33].

To obtain the optical pump rate $P_{i j}$, the equation of motion for the microscopic polarization $p_{i j}=\left\langle a_{i}^{\dagger} a_{j}\right\rangle$ is solved within the Markow and rotating-wave approximation. For a constant optical pump field with the frequency $\omega_{\mathrm{P}}$, intensity $I_{\mathrm{P}}$, and polarization $\mathbf{e}_{\mathrm{P}}$ one obtains

$$
\begin{aligned}
P_{i j}= & \left(\frac{e_{0}}{m_{0}}\right)^{2} \frac{\pi I_{\mathrm{P}}}{\epsilon_{0} c \omega_{\mathrm{P}}^{2}}\left|\mathbf{M}_{i j} \cdot \mathbf{e}_{\mathrm{P}}\right|^{2} \\
& \times\left(\mathcal{L}\left(\gamma_{i j}, \omega_{i j}+\omega_{\mathrm{P}}\right)+\mathcal{L}\left(\gamma_{i j}, \omega_{i j}-\omega_{\mathrm{P}}\right)\right) .
\end{aligned}
$$

The degeneracy of Landau levels in spin $s= \pm 1 / 2$, valley $\xi= \pm 1$, and quantum number $m=0,1, \ldots, N_{B}-1$ gives rise to a total amount of $4 N_{B}$ LLs with the same energy. Our numerical calculations show that for $N_{B} \gg 1$ the electronic dynamics depend only on the Landau-level index $l$, i.e., all degenerated levels behave equally. Thus, we define averaged quantities,

$$
\begin{gathered}
\rho_{l}=\frac{1}{4 N_{B}} \sum_{m, s, \xi} \rho_{(l, m, s, \xi)}, \\
S_{l, l^{\prime}}^{\mu}=\frac{1}{4 N_{B}} \sum_{m, s, \xi} S_{(l, m, s, \xi)\left(l^{\prime}, m, s, \xi\right)}^{\mu},
\end{gathered}
$$

where we have to consider only $s-, \xi$-, and $m$-diagonal polarizations, since other polarizations are forbidden by selection rules [19]. $T_{i}^{\mu}$ and $U_{i j}^{\mu}$ are treated in an analogous manner. As we assume that all observables are in good approximation independent of $m, s$, and $\xi$, we set $\rho_{l} \approx \rho_{(l, m, s, \xi)}, S_{l, l^{\prime}}^{\mu} \approx$ $S_{(l, m, s, \xi)\left(l^{\prime}, m, s, \xi\right)}^{\mu}$, and so forth. Hence, the photon generation rate in Eq. (A8) can be written as

$$
\sum_{i j}\left|g_{i j}^{\mu}\right|^{2} S_{j i}^{\mu} \approx 4 N_{B} \sum_{l_{i}, l_{j}}\left|g_{l_{i} l_{j}}^{\mu}\right|^{2} S_{l_{j} l_{i}}^{\mu},
$$

where [19] $g_{i j}^{\mu}=g_{l_{i} l_{j}}^{\mu} \delta_{m_{i}, m_{j}} \delta_{s_{i}, s_{j}} \delta_{\xi_{i}, \xi_{j}}$. The same procedure applies for the sums in Eq. (A12).

\section{Laser efficiency}

A figure of merit for a laser device is its power conversion efficiency, which for an optically driven laser is given by the intensity ratio of the incident pump field and the generated output. To calculate the output intensity of the Landau-level laser, we assume that the photon decay rate $2 \kappa$ [cf. Eq. (A8)] is determined solely by cavity-light out-coupling. In that case, the overall output intensity is given by $I_{\mathrm{Out}}=2 \kappa \sum_{\mu} \hbar \omega_{\mu} n_{\mu} / A$, i.e., the number of dissipated photons per time and cavity cross section $A$. Figure 6(a) shows the input-output curve for three exemplary magnetic fields at $T=300 \mathrm{~K}$ and $Q=5000$.

All three output characteristics show a strong nonlinear behavior due to saturation of the power conversion with increasing pump intensities. Such power characteristics are typical of three-level systems and result from finite pump and relaxation rates as discussed in the text. The laser threshold as well as the slope of the input-output curve (slope efficiency) [cf. Fig. 6(b)] is guided by the interplay of phonon relaxation channels, yielding a strong magnetic-field dependence of the power conversion rate. Our calculations reveal that even in the chosen exemplary model, based on one single graphene layer as the gain medium, $\mathrm{THz}$ output powers of several $\mathrm{W} / \mathrm{cm}^{2}$ can be achieved. The external efficiency $\eta_{\text {ext }}$ of the proposed laser design is essentially determined by the product

$$
\eta_{\text {ext }}=\eta_{\text {pump }} \times \eta_{\text {defect }} \times \eta_{\text {int }} \times \eta_{\text {cavity }},
$$

where $\eta_{\text {pump }}$ characterizes the pump absorption efficiency, $\eta_{\text {defect }}$ stands for the ratio of laser and pump frequency, $\eta_{\text {int }}$ reflects the internal quantum efficiency of the gain medium, 


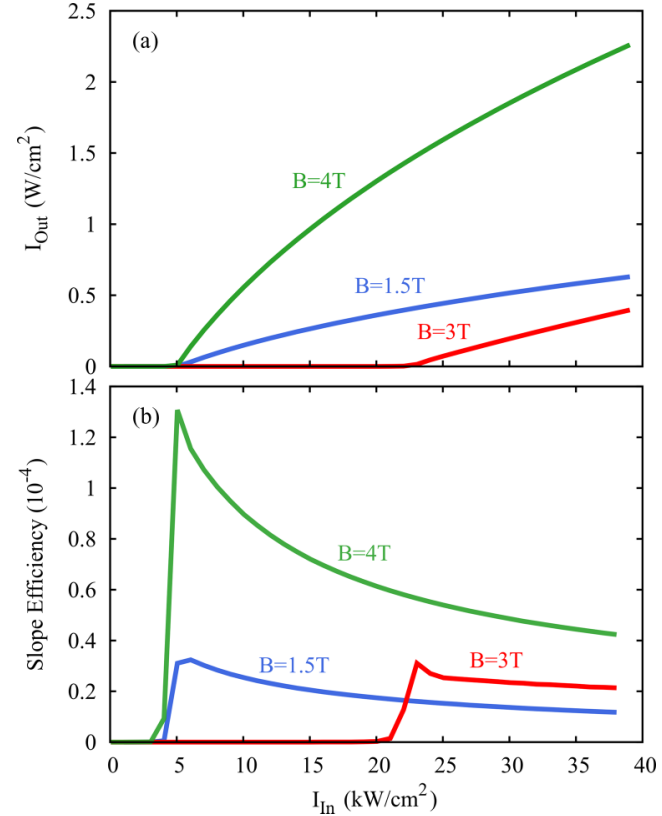

FIG. 6. Input-output characteristics. (a) THz output intensities of the Landau-level laser in relation to the incident pump intensity for three characteristic magnetic fields at $T=300 \mathrm{~K}$ and $Q=5000$ and (b) corresponding slope efficiencies $d I_{\text {Out }} / d I_{\text {In }}$.

and $\eta_{\text {cavity }}$ accounts for the out-coupling efficiency of the laser cavity. In our particular pump scheme, the energy defect due to the frequency conversion is given by $\eta_{\text {defect }}=(\sqrt{2}-$ $\sqrt{1}) /(\sqrt{3}+\sqrt{2}) \approx 0.13$, which is the upper limit for the efficiency of the proposed laser mechanism. The above-made assumption regarding the photon lifetime corresponds to a perfect out-coupling efficiency $\eta_{\text {cavity }}=1$, i.e., the only loss of cavity photons is given by the out-coupling of laser light. The comparatively low input/output ratio in the range of $10^{-5}-10^{-4}$ [cf. Fig. 6(b)] mainly results from the low absorption efficiency of our lasing setup, where only a small fraction $(\sim 1 \%)$ of the incident pump light is absorbed by the graphene layer. In a real laser device, the pump absorption efficiency could be significantly increased by using stacks of separated graphene layers and reusing unabsorbed pump light via mirrors.

The internal quantum efficiency of the Landau-level laser, i.e., the effective fraction of emitted laser photons per absorbed pump photon, is also in the range of several percent. This results from the Coulomb- and phonon-induced relaxation of excited carriers and from the parasitic absorption of cavity photons by the off-resonant transitions $l=2 \rightarrow 3, l=3 \rightarrow 4$, etc. The internal efficiency can be optimized by using dielectric substrates, which significantly screen the Coulomb scattering, or rather by providing lower operation temperatures to increase the Pauli blocking and therefore suppress unwanted phonon relaxation. The presence of several graphene layers as the gain medium would, in addition to better pump absorption, lead to an effective increase in the emitter density, which would yield an enhanced matter-light coupling and therefore would significantly improve the internal quantum efficiency.
[1] M. Tonouchi, Cutting-edge terahertz technology, Nat. Photon. 1, 97 (2007).

[2] E. Pickwell and V. P. Wallace, Biomedical applications of terahertz technology, J. Phys. D 39, R301 (2006).

[3] H.-B. Liu, H. Zhong, N. Karpowicz, Y. Chen, and X.-C. Zhang, Terahertz spectroscopy and imaging for defense and security applications, Proc. IEEE 95, 1514 (2007).

[4] J. Federici and L. Moeller, Review of terahertz and subterahertz wireless communications, J. Appl. Phys. 107, 111101 (2010).

[5] H. Aoki, Novel Landau level laser in the quantum Hall regime, Appl. Phys. Lett. 48559 (1986).

[6] T. Morimoto, Y. Hatsugai, and H. Aoki, Cyclotron radiation and emission in graphene-A possibility of Landau-level laser, J. Phys.: Conf. Ser. 150, 022059 (2009).

[7] F. Wendler and E. Malic, Towards a tunable graphene-based Landau level laser in the terahertz regime, Sci. Rep. 5, 12646 (2015).

[8] M. O. Goerbig, Electronic properties of graphene in a strong magnetic field, Rev. Mod. Phys. 83, 1193 (2011).

[9] M. L. Sadowski, G. Martinez, M. Potemski, C. Berger, and W. A. de Heer, Landau Level Spectroscopy of Ultrathin Graphite Layers, Phys. Rev. Lett. 97, 266405 (2006).

[10] K. M. Rao and J. E. Sipe, Coherent photocurrent control in graphene in a magnetic field, Phys. Rev. B 86, 115427 (2012).

[11] F. Wendler, A. Knorr, and E. Malic, Carrier multiplication in graphene under Landau quantization, Nat. Commun. 5, 3703 (2014).
[12] Y. Wang, M. Tokman, and A. Belyanin, Continuous-wave lasing between Landau levels in graphene, Phys. Rev. A 91, 033821 (2015).

[13] M. Engel, M. Steiner, A. Lombardo, A. C. Ferrari, H. v. Löhneysen, P. Avouris, and R. Krupke, Light-matter interaction in a microcavity-controlled graphene transistor, Nat. Commun. 3, 906 (2012).

[14] H. Haug and S. W. Koch, Quantum Theory of the Optical and Electronic Properties of Semiconductors, Vol. 5 (World Scientific, Singapore, 1990).

[15] F. Rossi and T. Kuhn, Theory of ultrafast phenomena in photoexcited semiconductors, Rev. Mod. Phys. 74, 895 (2002).

[16] M. Kira and S. Koch, Many-body correlations and excitonic effects in semiconductor spectroscopy, Prog. Quantum Electron. 30, 155 (2006).

[17] E. Malic and A. Knorr, Graphene and Carbon Nanotubes: Ultrafast Optics and Relaxation Dynamics (John Wiley \& Sons, New York, 2013).

[18] S. Reich, J. Maultzsch, C. Thomsen, and P. Ordejón, Tightbinding description of graphene, Phys. Rev. B 66, 035412 (2002).

[19] F. Wendler, A. Knorr, and E. Malic, Ultrafast carrier dynamics in Landau-quantized graphene, Nanophotonics 4, 224 (2015).

[20] E. Malic, T. Winzer, E. Bobkin, and A. Knorr, Microscopic theory of absorption and ultrafast many-particle kinetics in graphene, Phys. Rev. B 84, 205406 (2011). 
[21] S. Piscanec, M. Lazzeri, F. Mauri, A. C. Ferrari, and J. Robertson, Kohn Anomalies and Electron-Phonon Interactions in Graphite, Phys. Rev. Lett. 93, 185503 (2004).

[22] J. Maultzsch, S. Reich, C. Thomsen, H. Requardt, and P. Ordejón, Phonon Dispersion in Graphite, Phys. Rev. Lett. 92, 075501 (2004).

[23] T. Ando and Y. Uemura, Theory of quantum transport in a two-dimensional electron system under magnetic fields. I. Characteristics of level broadening and transport under strong fields, J. Phys. Soc. Jpn. 36, 959 (1974).

[24] N. H. Shon and T. Ando, Quantum transport in two-dimensional graphite system, J. Phys. Soc. Jpn. 67, 2421 (1998).

[25] H. Funk, A. Knorr, F. Wendler, and E. Malic, Microscopic view on Landau level broadening mechanisms in graphene, Phys. Rev. B 92, 205428 (2015).

[26] A. López, A. Di Teodoro, J. Schliemann, B. Berche, and B. Santos, Laser-induced modulation of the Landau level structure in single-layer graphene, Phys. Rev. B 92, 235411 (2015).
[27] Y. A. Bychkov and G. Martinez, Magnetoplasmon excitations in graphene for filling factors $v \leqslant 6$, Phys. Rev. B 77, 125417 (2008).

[28] M. O. Scully and M. S. Zubairy, Quantum Optics (Cambridge University Press, Cambridge, UK, 1997).

[29] C. Gies, J. Wiersig, M. Lorke, and F. Jahnke, Semiconductor model for quantum-dot-based microcavity lasers, Phys. Rev. A 75, 013803 (2007)

[30] R. Jago, T. Winzer, A. Knorr, and E. Malic, Graphene as gain medium for broadband lasers, Phys. Rev. B 92, 085407 (2015).

[31] K. J. Vahala, Optical microcavities, Nature 424, 839 (2003).

[32] T. Chen, P. Liu, J. Liu, and Z. Hong, A terahertz photonic crystal cavity with high $q$-factors, Appl. Phys. B 115, 105 (2014).

[33] M. Mittendorff, F. Wendler, E. Malic, A. Knorr, M. Orlita, M. Potemski, C. Berger, W. A. de Heer, H. Schneider, M. Helm et al., Carrier dynamics in Landau-quantized graphene featuring strong Auger scattering, Nat. Phys. 11, 75 (2015). 